\title{
How to Select the Pilot Carrier Positions in CP-OFDM?
}

\author{
Heidi Steendam \\ DIGCOM research group, TELIN Dept., Ghent University \\ Sint-Pietersnieuwstraat 41, 9000 GENT, BELGIUM \\ E-mail: Heidi.Steendam@telin.ugent.be
}

\begin{abstract}
In this paper, we consider the problem of pilot aided channel estimation (PACE) for CP-OFDM based systems. The positions of the pilot carriers have a strong influence on the mean squared error (MSE) of the channel estimate, and thus indirectly on the overall system performance. Hence, we are interested in the pilot carrier placement that minimizes this MSE. However, the optimization problem at hand is an integer combinatorial optimization problem and thus NP hard. In this paper, we propose two heuristic algorithms searching for a good pilot carrier placement with a low MSE. Both algorithms have comparable performance: the resulting MSE is for both algorithms similar, and in the majority of the cases the optimal pilot carrier placement is found. We compare the results from the proposed heuristic algorithms with the results for the maximum distance distribution from [1]. We are able to achieve lower channel estimate MSE than with the maximum distance distribution, although the difference becomes small for large FFT size.
\end{abstract}

\section{INTRODUCTION}

Many standards rely on the orthogonal frequency division multiplexing (OFDM) technique to cope with channel dispersion [2]. The structure of the OFDM system involves that the channel is split into many narrowband subchannels, such that equalization becomes a rather simple task. However, this requires the channel to be known. Since the advent of OFDM, much research has been devoted to channel estimation (see e.g. [3]). Usually, to estimate the channel, pilot carriers are sparsely inserted in the time-frequency grid of the OFDM signal [4], and based on the observations of these pilot carriers, the channel on the other (data) carriers is estimated. This can be achieved by interpolation [5]-[7] or by assuming an underlying channel model [8]. As in contrast to the interpolation approach, the assumption of a channel model does not lead to a performance error floor, we concentrate on this latter approach.

In many practical situations, this underlying channel model can assumed to be sparse, in the sense that the channel is modelled by a small number of channel taps. The observation of the pilot carriers is used to estimate these channel taps, from which the channel response at the intermediate carriers can be computed straightforwardly. It turns out that the performance of this technique depends on the positions of the pilot carriers. In the literature, several papers can be found dealing with the placement of the pilot carriers. In [9], the authors considered the special case where the number $M$ of pilot carriers divides the total number $N$ of carriers, and found that equispaced pilots are optimal. Similar results were found in [10] and
[11]. However, when $N / M$ is not integer, the equispaced distribution of the pilots is no longer optimal. In [1] and [11], ad hoc solutions to the pilot positions problem were proposed for $N / M$ not integer; the maximum distance distribution from [1] reduces to the ad hoc distribution from [11] for the special case of no guard bands or null carriers, where it is not allowed to place pilot carriers. Other researchers have tried to find a good pilot carrier placement by optimizing some cost function [12]-[15]. Common to all these techniques is that none of the proposed techniques achieves the minimum MSE of the channel estimate. Further, except for [1], [8], [14] - [15] these contributions do not take into account the presence of guard bands or null carriers.

In this paper, our goal is to find the optimal pilot carrier placement that minimizes the MSE. However, this optimization problem is NP hard, such that an exhaustive search is prohibitively complex. For example, for an FFT size of 32, it took already several weeks to obtain the optimal set of pilot carrier positions on a standard computer per parameter set. To find a close to optimum solution, we propose two heuristic search algorithms, one based on branch and bound (B\&B) [16] and one based on hill climbing (HC) local optimization [17]. For small values of the FFT size, we have compared the results from the two algorithms with the results from an exhaustive search, and observed that in the majority of the cases the two algorithms found the optimal pilot carrier placement (in $97.4 \%$ and $87.2 \%$ of the cases with branch and bound, and hill climbing, respectively). In the other cases, the resulting MSE was very close to the optimum, for both algorithms. For larger values of the FFT size, where an exhaustive search was not possible, the results from the two algorithms were compared with a theoretical lower bound on the MSE. The MSEs resulting from the two algorithms are very close to this theoretical lower bound, indicating that the two algorithms are virtually able to achieve the optimum MSE. A comparison of the computational complexity of the two algorithms shows that the hill climbing algorithm has lower complexity than the branch and bound algorithm, but the branch and bound algorithm results in (slightly) lower MSE. With both algorithms, we are able to outperform the low cost reference maximum distance distribution from [1] 1. The difference in MSE, however, decreases with increasing

\footnotetext{
${ }^{1}$ The maximum distance distribution that is introduced in [1] maximizes the distance between adjacent pilot carriers taking into account the presence of null edge subcarriers.
} 
FFT size $N$, indicating that for large $N$, the maximum distance distribution is an acceptable suboptimal solution. However, for smaller FFT sizes, using (one of) the proposed algorithms is recommended to obtain the pilot carrier positions with lowest MSE.

\section{The Multicarrier System}

In this paper, we assume that the channel impulse response does not change during an OFDM interval. As a result, we can model the channel as a tapped delay line $h(k), k=$ $0, \ldots, L-1$, with $L$ the length of the channel. Further, we assume the absence of intersymbol interference, i.e. the cyclic prefix length $\nu$ is larger than the channel impulse response duration: $\nu \leq L-1$. We insert $M \geq L$ pilots in the frequency domain at carrier positions $n_{\ell}, \ell=1, \ldots, M$ to estimate the unknown channel taps. At the receiver side, the $M$ FFT outputs corresponding to the pilot carriers can be written as

$$
\mathbf{y}=\mathbf{A h}+\mathbf{w}
$$

where $\mathbf{A}(\ell, k)=\sqrt{\frac{N}{N+\nu}} a_{p}(\ell) e^{-j 2 \pi \frac{k n_{\ell}}{N}}, \ell=1, \ldots, M, k=$ $0, \ldots, L-1, a_{p}(\ell)$ are the pilot symbols and $\mathbf{w}$ is the additive Gaussian noise. The ML estimate of the channel vector is given by

$$
\hat{\mathbf{h}}_{M L}=\left(\mathbf{A}^{H} \mathbf{A}\right)^{-1} \mathbf{A}^{H} \mathbf{y}
$$

and the MSE of the estimate yields

$$
M S E=E\left[\left\|\hat{\mathbf{h}}_{M L}-\mathbf{h}\right\|^{2}\right]=N_{0} \operatorname{trace}\left(\left(\mathbf{A}^{H} \mathbf{A}\right)^{-1}\right) .
$$

The MSE depends on the pilot carrier positions. Tests show that, when these pilot carrier positions are not well chosen, the matrix $\mathbf{A}^{H} \mathbf{A}$ can become (close to) singular, resulting in a very high MSE, and failing channel estimation.

Let us look closer to the matrix product $\mathbf{A}^{H} \mathbf{A}$. Assuming that $\left|a_{p}(\ell)\right|^{2}=E_{s}$, this matrix product can be written as

$$
\left(\mathbf{A}^{H} \mathbf{A}\right)_{k, k^{\prime}}=E_{s} \frac{N}{N+\nu} \sum_{\ell=1}^{M} e^{j 2 \pi \frac{\left(k-k^{\prime}\right) n_{\ell}}{N}} .
$$

Defining the Hermitian Toeplitz matrix B with elements $\mathbf{B}_{k, k^{\prime}}=b_{k-k^{\prime}}$, where

$$
b_{k}=\sum_{\ell=1}^{M} e^{j 2 \pi \frac{k n_{\ell}}{N}},
$$

the MSE can be rewritten as

$$
M S E=\left(\frac{E_{s}}{N_{0}}\right)^{-1} \frac{N+\nu}{N} \operatorname{trace}\left(\mathbf{B}^{-1}\right) .
$$

It can easily be verified that, if there were no limitations on $n_{\ell}$ (in reality $n_{\ell}$ is an integer $\in S_{c}$, where $S_{c} \cup S_{p}=\{0: N-1\}$, with $S_{c}$ the set of potential pilot carrier positions and $S_{p}$ the set of forbidden carrier positions) and thus on $b_{k}$, the minimum MSE would be reached when all eigenvalues of $\mathbf{B}$ are equal. In that case, the matrix $\mathbf{B}$ reduces to a diagonal matrix, because, if we decompose $\mathbf{B}$ as $\mathbf{B}=\mathbf{Q}^{H} \mathbf{\Lambda} \mathbf{Q}$, where $\boldsymbol{\Lambda}=\lambda \mathbf{I}$ is the eigenvalue matrix with all eigenvalues equal, and $\mathbf{Q}$ is the orthogonal eigenvector matrix $\left(\mathbf{Q}^{H} \mathbf{Q}=\mathbf{I}\right)$, it follows that
$\mathbf{B}=\lambda \mathbf{I}$. Taking into account that $b_{0}=M$, the resulting value of the MSE would be

$$
M S E_{L B}=\frac{N+\nu}{N} \frac{L}{M}\left(\frac{E_{s}}{N_{0}}\right)^{-1} .
$$

However, as in general it is not possible to find a pilot carrier placement that diagonalizes the matrix $\mathbf{B}$, this is a theoretical lower bound on the MSE. In [11], it is shown that when $M$ is a power of 2 (given that $N$ is also a power of 2), the equidistant pilot distribution diagonalizes $\mathbf{B}$. This result can be generalized to the case where $M$ divides $N$ when $N$ is not restricted to be a power of 2 [10]. As such, for these special cases, the optimal distribution is known. When $M$ does not divide $N$, the optimal solution is not known. In this paper, we will use this lower bound to assess the performance of the two heuristic search algorithms described in the next section.

\section{Heuristic SeArch Algorithms}

\section{A. Hill climbing $(\mathrm{HC})$}

The hill climbing (HC) technique [17] is a local search optimization technique that starts from an initial choice for the settings, and then looks in the neighbourhood, by incrementally changing a single element of the settings, to see if there is a solution that is better than the current one. If the function to be optimized has multiple local optima, the algorithm typically will get stuck in a local optimal point and the global optimum will easily be missed. Hence, the performance of this algorithm strongly depends on the initial settings. Usually, the HC algorithm is restarted multiple times with different (random) initializations to solve the problem of the local optima.

The hill climbing algorithm we propose is given in table I. In this algorithm, we first select the initial pilot carrier positions $n_{\ell}, \ell=1, \ldots, M$, where the carrier positions must be selected out of the set $S_{c}$ of possible positions (excluding the null subcarriers and guard band carriers). Then, we systematically switch one of the $M_{d}$ data carrier positions with a pilot carrier position, and determine if the resulting MSE is lower than the current best MSE. Unfortunately, the optimization problem at hand has many local minima. To stand a fair chance of finding the global minimum, the initial setting must therefore be close to the global optimum. Hence, the main problem in this algorithm is to find a good initialization. In our simulations, we used the maximum distance distribution from [1] as the initial pilot carrier placement. The convergence of the algorithm can be improved by iteratively executing the algorithm, where the output of the previous run is taken as the initial setting of the next run. Simulations have shown that typically one to five iterations are necessary before the algorithm shows no further improvement.

The proposed algorithm is a greedy algorithm: for all possible incremental changes the MSE must be computed. The computational load is dominated by the inversion of an $L \times L$ matrix, which has complexity $\mathcal{O}\left(L^{3}\right)$, such that the computational complexity per run of the algorithm equals $\mathcal{O}\left(M \cdot M_{d} \cdot L^{3}\right)$. This results in a computation time per run of some seconds for small $N$ to a couple of minutes for $N=1024$. 
TABLE I

HILL CLIMBING ALGORITHM

select initial pilot carrier positions $\mathbf{n}=\left\{n_{\ell} \mid \ell=1: M\right\}, n_{\ell} \in S_{c}$

determine $M_{d}$ data carrier positions $\tilde{\mathbf{n}}=S_{c} \backslash \mathbf{n}$

compute $M S E$

for $i=1: M$

for $j=1: M_{d}$

$\mathbf{n}^{\prime}=\mathbf{n} ; \tilde{\mathbf{n}}^{\prime}=\tilde{\mathbf{n}}$

$\mathbf{n}^{\prime}(i)=\tilde{\mathbf{n}}(j) ; \tilde{\mathbf{n}}^{\prime}(j)=\mathbf{n}(i)$

compute $M S E^{\prime}$ for $\mathbf{n}^{\prime}$

if $M S E^{\prime}<M S E$

$\mathbf{n}=\mathbf{n}^{\prime} ; \tilde{\mathbf{n}}=\tilde{\mathbf{n}}^{\prime} ; M S E=M S E^{\prime}$

end

end

end

\section{B. Branch and Bound $(B \& B)$}

The main problem with the hill climbing algorithm is the need for a good initial choice for the pilot carrier positions. Tests for small $N$, where the determination of the optimal pilot carrier placement through exhaustive search was still possible within reasonable time, revealed that in only $87.2 \%$ of the cases, the optimal pilot carrier placement was found with the hill climbing algorithm. In almost $13 \%$ of the cases, the maximum distance distribution was not close enough to the optimal distribution such that the hill climbing algorithm gets stuck in a local optimum. In this section, we consider another heuristic algorithm that does not suffer from bad initializations and local minima.

First let us look closer at the optimization problem at hand. The optimal pilot carrier placement minimizes the MSE from (6):

$\arg \min _{n_{\ell} \in S_{c}}\left(\frac{E_{s}}{N_{0}}\right)^{-1} \frac{N+\nu}{N} \operatorname{trace}\left(\mathbf{B}^{-1}\right)=\arg \min _{n_{\ell} \in S_{c}} \operatorname{trace}\left(\mathbf{B}^{-1}\right)$.

This involves the computation of the trace of the inverse of the Hermitian Toeplitz matrix $\mathbf{B}$, where the elements of $\mathbf{B}$ depend in a non-linear way on the pilot carrier positions to be optimized. Defining the eigenvalues $\lambda_{m}, m=1, \ldots, L$ of the matrix $\mathbf{B}^{2}$, trace $\left(\mathbf{B}^{-1}\right)$ can be rewritten as $\operatorname{trace}\left(\mathbf{B}^{-1}\right)=$ $\sum_{m=1}^{L} \frac{1}{\lambda_{m}}$. Assuming no restrictions on $\lambda_{m}$, this function is minimized when $\lambda_{m}=M$, i.e. all eigenvalues are equal. As the function $f(x)=\frac{1}{x}$ is a convex function for $x>0$ (i.e. the second derivative $f^{\prime \prime}(x)>0$ ), it follows that this minimum corresponds to a global minimum. Hence, the optimum pilot carrier placement will result in a matrix $\mathbf{B}$ with eigenvalues close to $\lambda_{m}=M$, which indicates that the matrix $\mathbf{B}$ is a close to diagonal matrix. As such, we are going to reformulate the optimization problem, such that it can be implemented with linear programming techniques.

As for the optimal pilot carrier placement the matrix $\mathbf{B}$ will be close to a diagonal matrix, its off diagonal elements will have small amplitudes. Therefore, taking into account that $\mathbf{B}$ is Hermitian Toeplitz, we reformulate the optimization problem

${ }^{2}$ These eigenvalues are real-valued because of the Hermitian nature of $\mathbf{B}$.
(8) into another related optimization problem:

$$
\arg \min _{n_{\ell} \in S_{c}} \sum_{k=1}^{L-1}\left|b_{k}\right|^{2}
$$

i.e. in (9), only the first row (column) of $\mathbf{B}$ is considered. Minimization of the off diagonal elements in the other rows (columns) will have a similar structure as (9), as most of the elements in the summation are the same. Taking into account that $\mathbf{B}$ is Toeplitz, the minimization of the sum of the squared moduli of the off diagonal elements (9) will guarantee that the matrix $\mathbf{B}$ will be close to diagonal. Note that the two optimization problems (8) and (9) are not equivalent. Hence, the solution from (9) will most probably not minimize (8). However, using the optimization problem (9), we will generate a restricted set of test pilot placements, out of which the one will be selected that minimizes the MSE (8). In the following we will show that the optimization problem (9) is a convex optimization problem that can be implemented by a branch and bound $(\mathrm{B} \& \mathrm{~B})$ algorithm, that belongs to the class of linear programming algorithms.

Let us take a closer look at the minimization (9):

$$
\begin{aligned}
\arg \min _{n_{\ell} \in S_{c}} \sum_{k=1}^{L-1}\left|b_{k}\right|^{2} & =\arg \min _{n_{\ell} \in S_{c}} \sum_{k=0}^{L-1}\left|b_{k}\right|^{2} \\
& =\arg \min _{n_{\ell} \in S_{c}} \sum_{k=0}^{L-1}\left|\sum_{\ell=1}^{M} e^{j 2 \pi \frac{k n_{\ell}}{N}}\right|^{2} \\
& =\arg \min _{n_{\ell} \in S_{c}} \sum_{\ell, \ell^{\prime}=1}^{M} g\left(n_{\ell}-n_{\ell^{\prime}}\right) \\
& =\arg \min _{n_{\ell} \in S_{c}} \sum_{\ell=1}^{M} \sum_{\ell^{\prime}=1}^{\ell-1} g\left(n_{\ell}-n_{\ell^{\prime}}\right)
\end{aligned}
$$

where $b_{k}$ is given by (5) and

$$
g(x)=\sum_{k=0}^{L-1} e^{j 2 \pi \frac{k x}{N}}=\cos \frac{\pi(L-1) x}{N} \cdot \frac{\sin \frac{\pi L x}{N}}{\sin \frac{\pi x}{N}}
$$

is a symmetric function: $g(-x)=g(x)$ and $g(N-x)=g(x)$. In (10a), we have extended the summation range (this has no influence as $b_{0}=M$ is a constant), and in (10b), we have substituted $b_{k}(5)$. In (10c), (11) is used to rewrite (10b), and in (10d), we made use of the symmetry of $g(x)$ to reduce the number of terms in the summation. The optimization problem (10d) can be implemented with the B\&B algorithm shown in table II. In this algorithm, we generate different candidate sets of pilot carrier positions. In the initialization phase, we define for each $n_{1} \in S_{c}$ a different candidate set, and gradually add extra pilot carrier positions to each set. At level $i$, for each of the (up to) $K_{M}$ sets $\left\{n_{1}, \ldots, n_{i-1}\right\}$, we add the $i$ th pilot carrier position $n_{i}$. The selection of the pilot carrier position $n_{i}$ is based on the branch metric

$$
f_{i}(y)=\sum_{\ell=1}^{i-1} g\left(y-n_{\ell}\right)
$$

that depends on the pilot carrier positions that are already in the considered set. For each of the sets, we select the $K_{g}$ pilot 
TABLE II

BRANCH\&BOUND ALGORITHM

compute $\tilde{g}(m), m=1, \ldots, N$

initialize tree $=\left[n_{1}, \Sigma_{1}=0\right], n_{1} \in S_{c}$

for $i=2: M$

treenew=empty; $x=\#$ rows in tree

for $k=1: x$

$\left\{n_{1}, \ldots, n_{i-1}\right\}=$ tree $(k ; 1: i-1)$

define $f_{i}(y)=\sum_{\ell=1}^{i-1} \tilde{g}\left(y-n_{\ell}\right), y \in S_{c}$

select the $K_{g}$ values with smallest $f_{i}\left(n_{i}^{j}\right)$

with $n_{i}^{j} \notin\left\{n_{1}, \ldots, n_{i-1}\right\}, j=1, \ldots, K_{g}$

for $j=1: K_{g}$

test if $\left\{n_{1}, \ldots, n_{i-1}, n_{i}^{j}\right\} \subset$ tree

if not: add pilot carrier position

$\Sigma_{i}=\Sigma_{i-1}+f\left(n_{i}^{j}\right)$; end

$$
\text { end }
$$

end

sort treenew on $\Sigma_{i}$ and select $K_{M}$ best ones to replace tree end

19) compute $M S E$ for all entries in tree and select the smallest

carrier positions $n_{i}$ with smallest branch metric, and generate the sets $\left\{n_{1}, \ldots, n_{i-1}, n_{i}^{j}\right\}, j=1, \ldots, K_{g}$. This results in (up to) $K_{g} \cdot K_{M}$ candidate sets at the next level. Hence, if we would keep all candidate sets, the computational load would increase exponentially. To reduce this computational load, we reduce the number of candidate sets again to $K_{M}$. The selection of the best candidate sets is based upon the cumulative metric, which is defined as

$$
\Sigma_{i}=\Sigma_{i-1}+f_{i}\left(n_{i}\right) .
$$

where $\Sigma_{1}=0$ : the $K_{M}$ sets with lowest cumulative metric are kept for the next level.

The algorithm requires three parameters to be selected: $K_{g}, K_{M}$ and $\alpha$. The first parameter $K_{g}$ limits the growth of the number of candidate sets within a level: the number of candidate sets grows (maximally) with a factor $K_{g}$. This factor can in reality be smaller than $K_{g}$ because we eliminate duplicate candidate sets to increase the convergence speed: if the set $\left\{n_{1}, \ldots, n_{i-1}, n_{i}^{j}\right\}$ is already present in the list of candidate sets, this new set is not kept for further processing. The second parameter $K_{M}$ limits the total number of candidate sets that are further processed and has also a large impact on the convergence of the algorithm: from the up to $K_{g} \cdot K_{M}$ candidate sets at each level, we keep only the $K_{M}$ sets with the lowest cumulative metrics. When $K_{M}$ is selected too small, there is a risk that the best pilot carrier placement is not found, whereas increasing $K_{M}$ increases the computational load of the algorithm. The algorithm outputs $K_{M}$ candidate sets out of which the one with the best MSE must be selected. It turns out that the convergence of the algorithm can be improved by introducing a new cost function $\tilde{g}(m)$

$$
\tilde{g}(m)=\operatorname{sign}(g(m)) \cdot|g(m)|^{\alpha}
$$

with $\alpha \in \mathbb{R}_{>0}$, i.e. $\alpha$ is positive real valued. In this way, we artificially adapt the shape of the function $g(m)$, to increase the difference between the branch and cumulative metrics of the different sets and obtain a better discrimination between the different carrier positions that can be added at a level, so

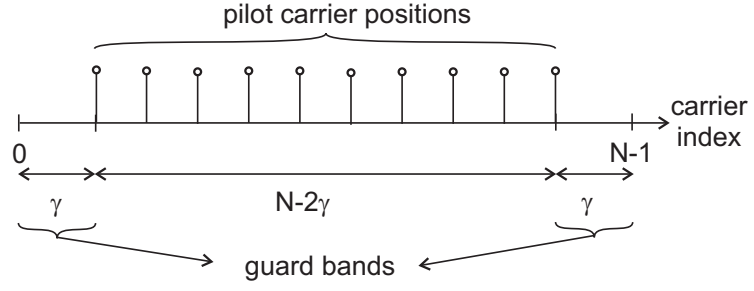

Fig. 1. Position of the guard bands.

that a smaller value for $K_{M}$ for the algorithm to converge is required.

The selection of the three parameters of the algorithm is not straightforward. In the simulations, we have searched for the optimal parameters that resulted in the minimum MSE. With this approach, the optimum pilot carrier placement determined with the exhaustive search was found in $97.4 \%$ of the cases. However, our approach required three one dimensional searches, the first one over $K_{g}$, where $K_{M} \approx 10 N$ and $\alpha=1$, the second one over $\alpha$, with the optimal value for $K_{g}$ and $K_{M} \approx 10 N$, and then increasing $K_{M}$ for the optimal values for $K_{g}$ and $\alpha$ until no further improvement on the MSE was found. However, from the simulations, it followed that in many cases the following choice for the parameters resulted in satisfactory MSE: $K_{g}=2-5, \alpha=1$ and $K_{M}=10-100 \times N$.

The computational complexity of the algorithm is mainly determined by the construction of the list of candidate sets, and is of the order of $\mathcal{O}\left((M-1) K_{g} K_{M} \log _{2} K_{g} K_{M}\right)$. This resulted in a computation time of a few seconds for small $N$ to half an hour for $N=1024$ using Matlab on a standard Windows computer $(2.54 \mathrm{GHz})$. Hence, the computational complexity is higher than the hill climbing algorithm, but the $\mathrm{B} \& \mathrm{~B}$ algorithm results in lower MSE and the optimum pilot carrier positions are found in more cases.

\section{NumericAl Results}

For the simulations, the guard band locations shown in figure 1 were considered. There are two guard bands of $\gamma$ carriers at the edges of the OFDM frequency band. Hence, there are $2 \gamma$ carriers that cannot be used for data transmission and pilot carrier placement. An example of the pilot carrier placement according to the maximum distance distribution and the hill climbing algorithm is shown in figure 2. For the maximum distance distribution, we can clearly see that the distance between adjacent pilot carriers is maximized. In the best pilot carrier placement found with the hill climbing algorithm, we observe that the best pilot carrier positions can strongly differ from the maximum distance distribution. Generally, the best pilot carrier positions found with the hill climbing algorithm or the branch\&bound algorithm will not show a regular pattern.

In figure 3, a comparison is made between the MSE corresponding to the maximum distance (MD) pilot carrier placement, and the MSE corresponding to the pilot carrier placements obtained with the hill climbing (HC) and branch and bound $(\mathrm{B} \& \mathrm{~B})$ algorithm, respectively. Two cases are considered: the case where there are no forbidden guard bands 


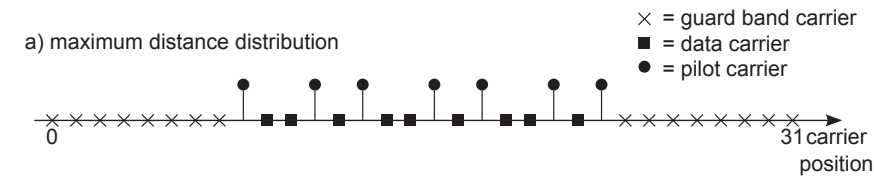

b) optimal pilot positions according to hill climbing algorithm

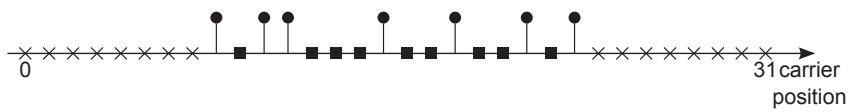

Fig. 2. Pilot carrier placement according to (a) the maximum distance distribution (b) the hill climbing algorithm for $N=32, M=7, \gamma=8$.

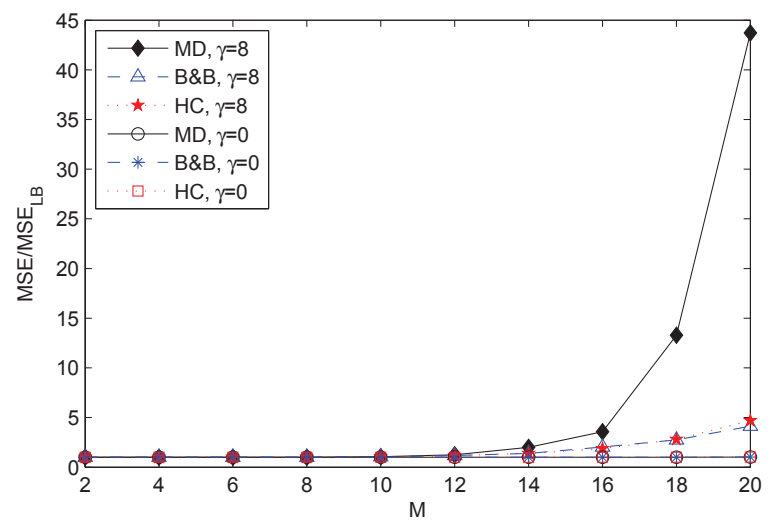

Fig. 3. Comparison of the MSE for maximum distance (MD) distribution and the distributions from the branch and bound (B\&B) algorithm and the hill climbing (HC) algorithm as function of $M, N=128, L=M$.

$(\gamma=0)$, i.e. the whole frequency band can be used for pilot carrier placement, and the case of two guard bands of 8 carriers, i.e. $\gamma=8$. As can be observed, for $\gamma=0$ the MSE normalized to its lower bound is very close to one, for all considered cases. Hence, when there are no guard bands, the maximum distance distribution is virtually able to reach the theoretical lower bound, and the use of the heuristic algorithms is not necessary. However, when $\gamma=8$ and for increasing $M$, there is a clear difference between the MSE for the maximum distance (MD) distribution and the MSE for the two heuristic algorithms. The MSE of the B\&B algorithm is slightly lower than the one for the $\mathrm{HC}$ algorithm, but the difference is very small. We observe that the MD distribution is far from optimal when there are guard bands present and the number of pilot carriers increases. With the proposed heuristic search algorithms, on the other hand, we are able to obtain pilot carrier placements that have a MSE close to the theoretical lower bound.

In figure 4, the effect of the guard band size $\gamma$ is evaluated. When $\gamma$ increases, the MSE will increase. This is anticipated, as with increasing guard band size, the number of possible pilot carrier positions decreases. Because of the lower flexibility in placing the pilot carriers, it will become more difficult to find a pilot carrier placement that 'diagonalizes' the matrix $\mathbf{B}$, i.e. the off diagonal elements will have larger amplitude. As a result, the MSE will increase. As in the previous figure, the two heuristic search algorithms result in an MSE that

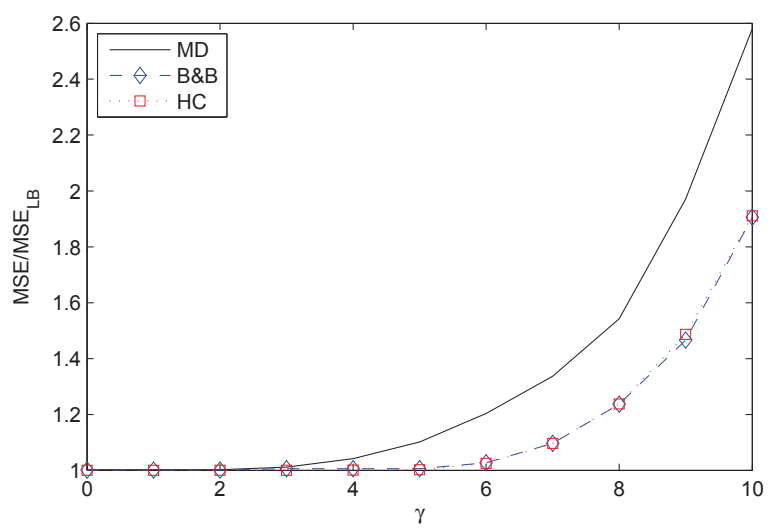

Fig. 4. Comparison of the MSE for maximum distance (MD) distribution and the distributions from the branch and bound (B\&B) algorithm and the hill climbing (HC) algorithm as function of $\gamma, N=64, L=5, M=15$.

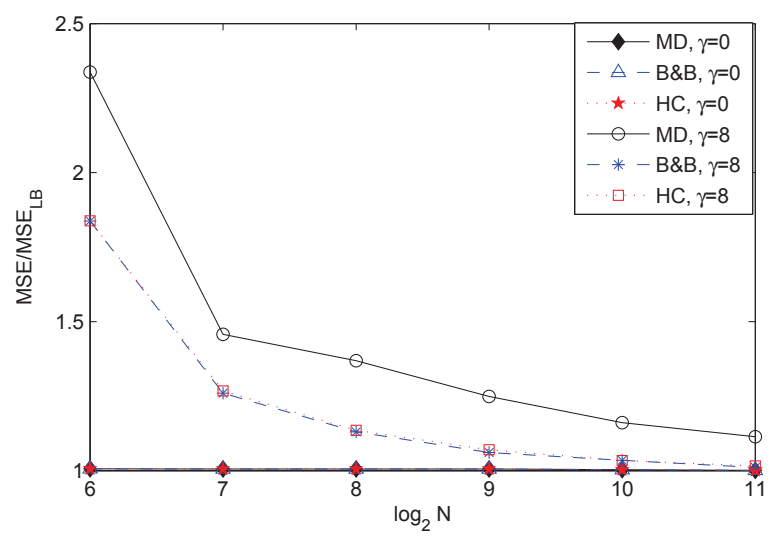

Fig. 5. Comparison of the MSE for maximum distance (MD) distribution and the distributions from the branch and bound (B\&B) algorithm and the hill climbing (HC) algorithm as function of $N, M=\lceil 0.1 \cdot N\rceil, L=M$.

is similar, and is clearly lower than that for the maximum distance distribution.

The effect of the FFT size $N$ is considered in figure 5 for $\gamma=0$ and $\gamma=8$. For $\gamma=0$, the MSE is very close to the theoretical lower bound, for all cases, whereas for $\gamma=8$, the difference is larger. However, in the latter case, the difference between the MSE and its lower bound decreases when the FFT size increases. This can be explained as when $N$ increases, the pilot carrier positions can be selected out of a larger set of carrier positions. Because of this higher flexibility, the matrix B can be better 'diagonalized'. Again, the two heuristic search algorithms have better performance than the MD distribution: for $N \geq 1024$, the resulting MSE is very close to the theoretical lower bound. Also the MSE for the MD distribution comes closer to the lower bound when $N$ increases: the MD distribution is therefore a good low cost suboptimal alternative for large $N$.

\section{CONCLUSIONS}

In this paper, we have proposed two heuristic search algorithms to find the pilot carrier placement that minimizes the MSE of the channel estimation in CP-OFDM. We have 
compared the results of the heuristic algorithms with the results of the maximum distance distribution from [1]. In the presence of guard bands, where the pilot carriers cannot be placed, the heuristic search algorithms result in clearly lower MSE than the MD distribution. Although the MD distribution turns out to be a good sub-optimal alternative for larger FFT size.

The question arises which of the proposed algorithms is best suited to determine the pilot carrier positions. On the one hand side, the branch and bound algorithm has better performance, as it does not get stuck in local minima as in the hill climbing algorithm. However, when comparing the results of the two algorithms, it turns out that in reality, the hill climbing algorithm has similar performance as the branch and bound algorithm: the resulting MSE is virtually the same. Hence, the main difference will lie in the complexity of the algorithm. As the hill climbing algorithm has lower complexity, and does not require parameters to be optimized as in the branch and bound algorithm, the hill climbing algorithm is more suited for practical use. Finally, for large $N(N>1024)$, the maximum distance distribution is a good suboptimal alternative that selects the pilot carrier positions at no computational cost.

\section{ACKNOWLEDGEMENT}

The author gratefully acknowledges the financial support from the Flemish Fund for Scientific Research (FWO). This research has been funded by the Interuniversity Attraction Poles Programme initiated by the Belgian Science Policy Office.

\section{REFERENCES}

[1] R.J. Baxley, J.E. Kleider, and G.T. Zhou, "Pilot Design for OFDM with Null Edge Subcarriers," IEEE Trans. on Wireless Communications, vol. 8, no. 1, pp. 396-405, Jan. 2009.

[2] L. Hanzo, M. Münster, B.J. Choi, T. Keller, OFDM and MC-CDMA for Broadband Multi-User Communications, WLANs and Broadcasting, Wiley, New York, 2003.

[3] M. Morelli and U. Mengali, "A Comparison of Pilot-Aided Channel Estimation Methods for OFDM Systems," IEEE Trans. Signal Process., vol. 49, no. 12, pp. 3065-3073, Dec. 2001.

[4] J.-W. Choi and Y.-H. Lee "Optimal Pilot Pattern for Channel Estimation in OFDM Systems," IEEE Trans. Wireless Commun., vol. 4, no. 5, pp. 2083-2088, Sep. 2005.

[5] H. Tang, K.Y. Lau, R.W. Brodersen, "Interpolation-Based Maximum Likelihood Channel Estimation Using OFDM Pilot Symbols," in Proc. Globecom 2002, pp. 1860-1864.

[6] O. Simeone, Y. Bar-Ness, U. Spagnolini, "Pilot-Based Channel Estimation for OFDM Systems by Tracking the Delay-Subspace," IEEE Trans. on Wireless Comm., vol. 3, no. 1, pp. 315-325, Jan. 2004.

[7] X. D. Dong, W.-S. Lu and A. C. K. Soong, "Linear Interpolation in Pilot Symbol Assisted Channel Estimation for OFDM," IEEE Transactions on Wireless Comm., vol. 6, no. 5, pp. 1910-1920, May 2007.

[8] S. Song, A.C. Singer, "Pilot-Aided OFDM Channel Estimation in the Presence of the Guard Band," IEEE Trans. on Comm., vol. 55, no. 8, pp. 1459-1465, Aug. 2007.

[9] R. Nehi, J.M. Cioffi, "Pilot Tone Selection for Channel Estimation in a Mobile OFDM System," IEEE Transactions on Consumer Electronics, vol. 44, no. 3, pp. 1122-1128, Aug. 1998.

[10] M. Dong, L. Tong, and B. M. Sadler, "Optimal Pilot Placement for Channel Tracking in OFDM," in Proc. MILCOM 2002, pp. 602-606.

[11] X. Cai, G.B. Giannakis, "Error Probability Minimizing Pilots for OFDM With M-PSK Modulation Over Rayleigh-Fading Channels," IEEE Trans. on Vehicular Technology, vol. 53, no. 1, pp. 146-155, Jan. 2004.
[12] P. Pakrooh, A. Amini, F. Marvasti, "OFDM pilot allocation for sparse channel estimation," EURASIP Journal on Advances in Signal Processing 2012, 2012:59, doi:10.1186/1687-6180-2012-59.

[13] M.D. Larsen, G. Seco-Granados, A.L. Swindlehurst, "Pilot Optimization for Time-Delay and Channel Estimation in OFDM Systems," in Proc. IEEE International Conference on Acoustics, Speech, and Signal Processing, ICASSP 2011, pp. 3564-3567.

[14] S. Ohno, E. Manasseh, M. Nakamoto, "Preamble and Pilot Symbol Design for Channel Estimation in OFDM Systems with Null Subcarriers," EURASIP Journal on Wireless Communications and Networking 2011 2011:2, doi:10.1186/1687-1499-2011-2.

[15] B.R. Hamilton, X. Ma, J.E. Kleider, R.J. Baxley, ”OFDM Pilot Design for Channel Estimation with Null Edge Subcarriers,' IEEE Trans. on Wireless Communications, vol. 10, no. 10, pp. 3145-3150, Oct. 2011.

[16] A. H. Land, A. G. Doig, "An Automatic Method of Solving Discrete Programming Problems," Econometrica 28 (3), 1960, pp. 497-520, doi: $10.2307 / 1910129$

[17] T. Weise, "Global Optimization Algorithms - Theory and Application," 2009, available online: http://www.it-weise.de/. 